\title{
Incidence of sleep problems and their mediating role on depression and anxious preoccupation in patients with resected, non-advanced cancer: data from NEOcoping study
}

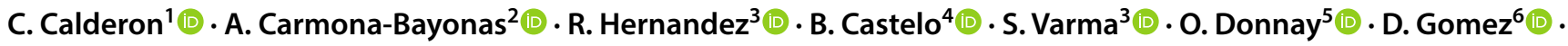 \\ P. Jimenez-Fonseca ${ }^{6}$ (D)
}

Received: 13 November 2018 / Accepted: 13 December 2018

(c) Federación de Sociedades Españolas de Oncología (FESEO) 2019

\begin{abstract}
Background Our study analyzes the incidence of sleep problems and their mediating role on depression and anxious preoccupation in patients with resected, non-advanced cancer.

Methods A multi-institutional, prospective, observational study was conducted with 750 participants of 14 hospitals in Spain. Participants' socio-demographic and clinical characteristics were collected using a standardized self-report form and using EORTC QoL-QLQ-C30, BSI, Mini-MAC questionnaires.

Results In women, sleep problems, depression and anxious preoccupation were observed in 65,41 and $21 \%$, respectively. In men, sleep problems, depression and anxious preoccupation were reported in 51, 29 and 61\%, respectively. More sleep problems, depression and anxious preoccupation were found among women than males. Depression was a significant predictor of anxious preoccupation. In males, sleep problems partially mediated this association. This was not confirmed in women. Conclusion Our findings point toward the importance of developing interventions that decrease depression and sleep problems in cancer.
\end{abstract}

Keywords Adjuvant chemotherapy · BSI · Coping $\cdot$ EORTC-QoL-QLQ-C30 $\cdot$ Mini-MAC $\cdot$ Quality of life

\section{Introduction}

Adjuvant chemotherapy following cancer resection increases survival in patients at risk for recurrence. High levels of anxious preoccupation often emerge during the pre-treatment period due to fear surrounding treatment side effects and recurrence [1]. Likewise, anxious preoccupation has been associated with depression and insomnia [2]. Similarly, depression is also common in oncological patients and can aggravate sleep disturbances [3] and together can contribute to anxious preoccupation, a coping strategy deemed inappropriate [4].

Sleep problems are prevalent in cancer patients [5], nearly two-thirds of patients reported symptoms including long latency periods before falling or staying asleep, frequent nocturnal awakenings, and impaired seep quality [6]. Higher levels of sleep problems predicted for more fatigue, depression and poorer performance status [7].

Few studies have examined anxious preoccupation in individuals about to receive chemotherapy, yet, in our series, it was present in $15.8 \%$ [1]. 
Our objective was to analyze the prevalence of depression, anxious preoccupation and sleep disorders in a population of patients with a resected non-advanced cancer grouped by gender and to know the mediational role of sleep problems in the other two disorders, depression and anxious preoccupation.

\section{Methods}

\section{Participants and procedures}

A multi-institutional, prospective, observational study was conducted with the participation of 14 hospitals in Spain from June 2015 to June 2018. NEOcoping is a cancer patient research program funded by the Continuous Care Group of the Spanish Society of Medical Oncology (SEOM). The study was approved by the Ethics Committee of each institution and by the Spanish Agency of Medicines and Medical Devices (AEMPS). Participants were 18 years of age or older with resected, non-advanced, histologically confirmed, solid cancer and eligible for adjuvant chemotherapy.

\section{Measures}

Participants' socio-demographic characteristics were collected using a standardized self-report form. Information related to participants' disease was obtained by reviewing their medical history.

Sleep problems: participant's subjective sleep was measured using the corresponding scale of a multidimensional questionnaire of health related quality of life (HRQoL), the EORTC QoL Questionnaire C30 (QLQ-C30) version 2.0 [8]. The questionnaire involves one straightforward question: 'during the past week, have you had trouble sleeping?' It was considered that patients had subjective sleep problems if they answered, 'a little', 'quite a bit' and 'very much', corresponding to $33.3,66.7$ or 100 values on the scale, respectively. This cut-off point was similar to that used by other authors [5]. In this study, Cronbach's alpha for the scale was 0.88 .

Depression was measured using the Brief Symptom Inventory (BSI) 6-item depression subscale [9], designed to assess symptoms of disaffection and dysphoric mood, e.g., those reflecting self-deprecation, anhedonia, hopelessness, and suicidal ideation. Raw scores were converted to $T$ scores [9]. In the present study, internal consistency was 0.75 .

\section{Anxious preoccupation}

The anxious preoccupation subscale of the Mini-Mental Adjustment to Cancer (Mini-MAC) scale was administered to quantify dysfunctional coping in response to cancer. It was taken from the Spanish version of the Mini-MAC [4] and consists of eight items (e.g., "I worry about the cancer returning or getting worse"), each measuring the tendency toward constant preoccupation with cancer as an event causing feelings of devastation, anxiety, fear, and apprehension. Responses above the 85th percentile (one standard deviation (SD) above the mean) were interpreted as clinically significant for high levels of anxious preoccupation. In this study, Cronbach's alpha for the scale range from 0.55 to 0.80 [10].

\section{Statistical analyses}

Chi-square and $t$ test were conducted to compare participants and no-participants' socio-demographic and clinic characteristics. Mediation analyses were performed to determine whether perceived stress mediated in the association of depression and anxious preoccupation-based coping in patients. Separate models were run for anxious preoccupation and path coefficients, and bootstrapped confidence intervals were estimated. Analyses were run on the IBM-SPSS 23.0 statistical software package for Windows PC.

\section{Results}

Of the 835 patients recruited, 750 were eligible and the other 85 did not meet the inclusion criteria or met at least some exclusion criteria. No significant intergroup differences were detected regarding socio-demographic and clinical baseline characteristics; see Table 1.

Sleep problems were observed in $65.5 \%$ of women and $51.1 \%$ of men. Reported sleep problems in women (median $(\mathrm{M})=36.4, \mathrm{SD}=33.9)$ was significantly higher than in men $(\mathrm{M}=25.1, \mathrm{SD}=29.6), t(748)=4.794, p<0.001$, $d=0.35-41.1 \%$ of women and $28.6 \%$ of men reported depression problems. There was significantly more perceived depression among women $(\mathrm{M}=61.5, \mathrm{SD}=6.0)$ than men $(\mathrm{M}=59.3, \mathrm{SD}=5.6, t(748)=-5.257), p<0.001$, $d=0.37$. Clinically significant scores in anxious preoccupation were displayed by $21.2 \%$ of women and $15.8 \%$ of men. More anxious preoccupation was also found among females $(\mathrm{M}=43.1, \mathrm{SD}=25.6)$ versus males $(\mathrm{M}=38.7, \mathrm{SD}=24.4$, $t(748)=-3.160), p<0.002, d=0.23$.

\section{Mediation analyses}

Depression was a significant predictor of anxious preoccupation (Fig. 1a). Among males, sleep problems partially mediated this association, confirmed by the significant, indirect effect of depression on sleep problems (indirect effect $=0.06$ : $95 \%$ confidence interval $=0.02-0.12$, Sobel $z=-3.023, p<0.002$ ). This was not confirmed in women (Sobel $z=1.129, p=0.258$ ) (Fig. 1b). 
Table 1 Socio-demographic and clinical characteristics of participants and nonparticipants

\begin{tabular}{lccc}
\hline Characteristics & Participants $(n=750)$ & $\begin{array}{l}\text { No-participants } \\
(n=85)\end{array}$ & $p$ value \\
\hline Age (mean; SD) & $59.7(12.2)$ & $58.4(11.1)$ & 0.444 \\
Gender $(n, \%$ female) & $434(57.9)$ & $68(75.0)$ & 0.233 \\
Marital status $(n, \%$ married or partnered) & $570(76.0)$ & $68(75.0)$ & 0.936 \\
Education (years, $n, \mathrm{SD})$ & $7.9(4.8)$ & $7.8(4.8)$ & 0.667 \\
Employment status $(n, \%$ inactive) & $450(60.0)$ & $61(67.0)$ & 0.640 \\
Tumor localization $(n, \mathrm{SD})$ & & $26(28.5)$ & 0.105 \\
Colon & $329(43.9)$ & $45(49.4)$ & \\
Breast & $235(31.3)$ & $20(21.9)$ & \\
Others & $186(24.8)$ & $53(58.2)$ & 0.738 \\
Tumor stage $(n, \%$ I-II) & $401(55.2)$ & $37(40.7)$ & 0.105 \\
Chemo- and radiotherapy $(n, \%)$ & $245(32.7)$ & & \\
\hline
\end{tabular}
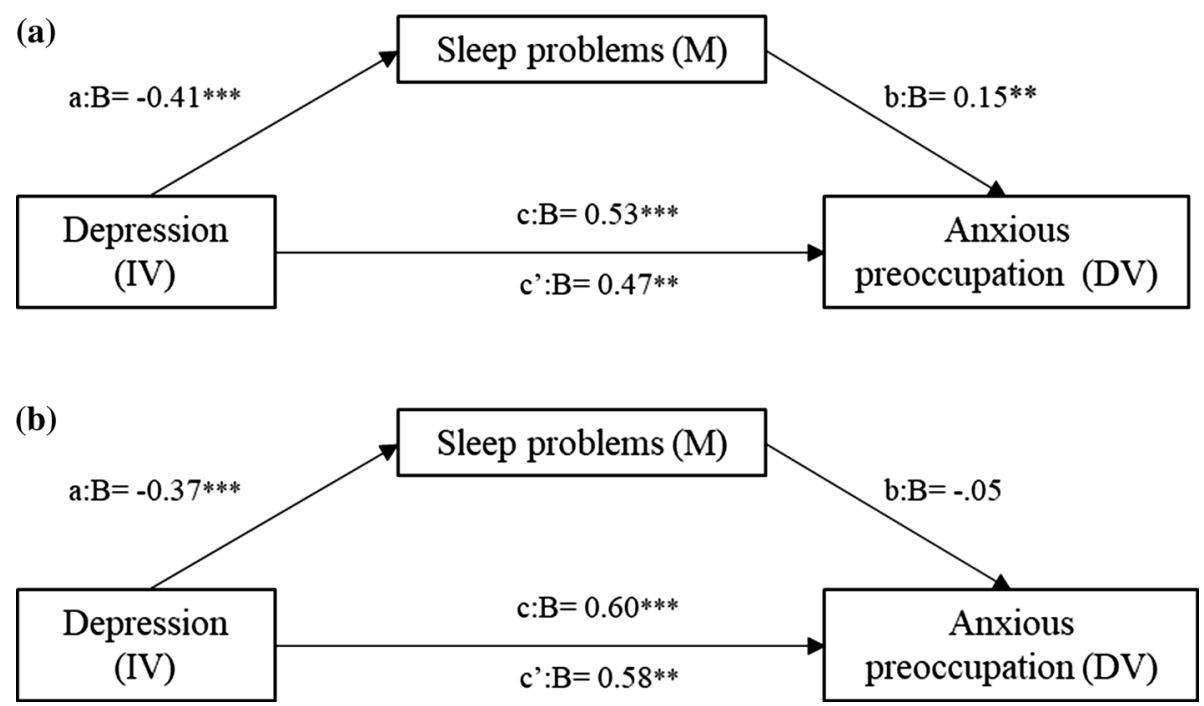

Fig. 1 Test of sleep problems as mediator of association between depression and anxious preoccupation. Mediation model showing that the effect of depression (independent variable) on anxious preoccupation (outcome) is mediated by sleep problems (mediator). Changes in Beta weights when the mediator is present are showed in c'. The result in the first step indicated that depression $(B=-0.53$ and $\mathrm{B}=0.60$ ) predicted anxious preoccupation. When sleep problems (mediator) were controlled the weight of depression was reduced over anxious preoccupation but it was still significant $(B=0.47$ and

\section{Discussion}

This study examines sleep problems in cancer patients who will initiate adjuvant chemotherapy and confirms its high prevalence in both women and men, $65.5 \%$ and $51.1 \%$, respectively. Sleep problems (experienced between $12.5 \%$ and $56 \%$ ) is one of the most prevalent symptom in cancer patients and survivors $[5,7,11]$. The possible causes of this wide range of prevalence may be due to demographic variables such as age, type of tumor, and type of treatment
$\mathrm{B}=0.58$ ). Sleep problems, as a mediator, predicted anxious preoccupation even when depression was controlled $(B=-0.15$ and $\mathrm{B}=-0.05$ ). Sleep problems improved the prediction of anxious preoccupation over-and-above the independent variable (depression). a, direct effect of independent variable (IV) on mediator (M); b, direct effect of $\mathrm{M}$ on dependent variable (DV); c, direct effect of IV on DV; c', indirect effect of IV on DV; B, beta weights. ${ }^{*} p<0.05$, ${ }^{* *} p<0.01$, $* * * p<0.001$

$[6,11]$. Sleep problems together with depression predicted poor QoL in colorectal patients, the largest group in this series [7].

In the present study, women presented more sleep problems, depression and anxious preoccupation than men. Similar to that found in other studies [12]. Breast cancer patients with a sleep problem were more likely to experienced complications and had longer hospital stays [13], and worse QoL [14].

This study also evaluates a mediation model that hypothesizes that sleep problems mediate the association between 
anxious preoccupation and depression. The model was confirmed in men, albeit not in women, indicating that the greater the depression, the more sleep problems leading to increased anxious preoccupation in men. This demonstrates the association between depression and negative consequences for physical and psychological health [15]. Among females, the non-confirmation of the mediation model would indicate that there are other variables that account for anxious preoccupation and should be contemplated in future studies, including fear of deterioration of body image, personality, perceived social and family support.

The strengths of this study include its prospective, multicenter design, and a large sample of patients with nonadvanced resected cancer. Its limitations include the modest effect size, use of self-reports that can lead to response biases such as social desirability, memory errors, etc., and the cross-sectional design that prevents directionality from being inferred.

Our findings point toward the importance of developing interventions that decrease depression and sleep problems in men with cancer. This may help them in their coping strategies with evidence-based approaches, such as cognitive and/or behavioral treatments that incorporate relaxation techniques. Future studies should investigate how these states evolve over time and incorporate objective measures of sleep quality.

Acknowledgements The authors would like to thank the investigators of the NEOcoping study, the Supportive Care Working Group of the Spanish Society of Medical Oncology (SEOM), Natalia Cateriano, Miguel Vaquero and IRICOM S.A. for the support of the website registry, and Priscilla Chase Duran for editing the manuscript.

Sources of funding This work was funded by the Spanish Society of Medical Oncology (SEOM) in 2015. The sponsor of this research has not participated in data collection, analysis, or interpretation, in writing the report, or in the decision to submit the article for publication.

\section{Compliance with ethical standards}

Conflict of interest The authors have declared no conflict of interest.

Ethical approval (research involving human participants and/or animals) The study has been performed in accordance with the ethical standards of the Declaration of Helsinki and its later amendments. This study is an observational, non-interventionist trial.

Informed consent Signed informed consent was obtained from all patients.

\section{References}

1. Jimenez-Fonseca P, Calderon C, Carmona-Bayonas A, Muñoz MM, Hernández R, Mut Lloret M, et al. The relationship between physician and cancer patient when initiating adjuvant treatment and its association with sociodemographic and clinical variables. Clin Transl Oncol. 2018;20(11):1392-9.

2. Bower JE. Behavioral symptoms in breast cancer patients and survivors: fatigue, insomnia, depression, and cognitive disturbance. J Clin Oncol. 2008;26(5):768-77.

3. Loh KP, Zittel J, Kadambi S, Pandya C, Xu H, Flannery M, et al. Elucidating the associations between sleep disturbance and depression, fatigue, and pain in older adults with cancer. J Geriatr Oncol. 2018;9(5):464-8.

4. Costa-Requena G, Gil F. The mental adjustment to cancer scale: a psychometric analysis in Spanish cancer patients. Psychooncology. 2009;18(9):984-91.

5. Innominato PF, Spiegel D, Ulusakarya A, Giacchetti S, Bjarnason GA, Lévi F, et al. Subjective sleep and overall survival in chemotherapy-naïve patients with metastatic colorectal cancer. Sleep Med. 2015;16(3):391-8.

6. Howell D, Oliver TK, Keller-Olaman S, Davidson JR, Garland S, Samuels C, et al. Sleep disturbance in adults with cancer: a systematic review of evidence for best practices in assessment and management for clinical practice. Ann Oncol. 2014;25(4):791-800.

7. Mota DD, Pimenta CA, Caponero R. Fatigue in colorectal cancer patients: prevalence and associated factors. Rev Lat Am Enferm. 2012;20(3):495-503.

8. Aaronson NK, Ahmedzai S, Bergman B, Bullinger M, Cull A, Duez NJ, et al. The European Organization for Research and Treatment of Cancer QLQ-C30: a quality-of-life instrument for use in international clinical trials in oncology. J Natl Cancer Inst. 1993;85(5):365-76.

9. Derogatis LR, Melisaratos N. The brief symptom inventory: an introductory report. Psychol Med. 1983;13(3):595-605.

10. Grassi L, Buda P, Cavana L, Annunziata MA, Torta R, Varetto A. Styles of coping with cancer: the Italian version of the MiniMental Adjustment to Cancer (Mini-MAC) scale. Psychooncology. $2005 ; 14(2): 115-24$.

11. Zhou ES, Clark K, Recklitis CJ, Obenchain R, Loscalzo M. Sleepless from the get go: sleep problems prior to initiating cancer treatment. Int J Behav Med. 2018;25(5):502-16.

12. Van Onselen C, Paul SM, Lee K, Dunn L, Aouizerat BE, West C, et al. Trajectories of sleep disturbance and daytime sleepiness in women before and after surgery for breast cancer. J Pain Symptom Manage. 2013;45(2):244-60.

13. Vin-Raviv N, Akinyemiju TF, Galea S, Bovbjerg DH. Sleep disorder diagnoses and clinical outcomes among hospitalized breast cancer patients: a nationwide inpatient sample study. Support Care Cancer. 2018;26(6): 1833-40.

14. İzci F, Sarsanov D, Erdogan Zİ, İlgün AS, Çelebi E, Alço G, et al. Impact of personality traits, anxiety, depression and hopelessness levels on quality of life in the patients with breast cancer. Eur $\mathbf{J}$ Breast Health. 2018;14(2):105-11.

15. Ohrnberger J, Fichera E, Sutton M. The relationship between physical and mental health: a mediation analysis. Soc Sci Med. 2017;195:42-9.

Publisher's Note Springer Nature remains neutral with regard to jurisdictional claims in published maps and institutional affiliations. 